\title{
Ionospheric currents estimated simultaneously from CHAMP satellite and IMAGE ground-based magnetic field measurements: a statistical study at auroral latitudes
}

\author{
P. Ritter ${ }^{1}$, H. Lühr ${ }^{1}$, A. Viljanen ${ }^{2}$, O. Amm ${ }^{2}$, A. Pulkkinen ${ }^{2}$, and I. Sillanpää2 \\ ${ }^{1}$ GeoForschungsZentrum Potsdam, Telegrafenberg, D-14473 Potsdam, Germany \\ ${ }^{2}$ Finnish Meteorological Institute Geophys. Res. Div., P.O.Box 503, FIN-00101 Helsinki, Finland \\ Received: 31 January 2003 - Revised: 28 May 2003 - Accepted: 23 June 2003 - Published: 1 January 2004
}

\begin{abstract}
One important contribution to the magnetic field measured at satellite altitude and at ground level comes from the external currents. We used the total field data sampled by the Overhauser Magnetometer on CHAMP and the horizontal magnetic field measurements of the IMAGE groundbased magnetometer network to study the ionospheric Hall current system in the auroral regions. For the CHAMP data a current model consisting of a series of lines and placed at a height of $110 \mathrm{~km}$ is fitted to the magnetic field signature sampled on the passage across the polar region. The derived current distributions depend, among others, on season and on the local time of the satellite track. At dawn/dusk the auroral electrojets can be detected most clearly in the auroral regions. Their intensity and location are evidently correlated with the $A E$ activity index. For a period of almost two years the results obtained from space and the currents determined from ground-based observations are studied. For the full IMAGE station array a newly-developed method of spherical elementary current systems (SECS) is employed to compute the 2-D equivalent current distribution, which gives a detailed picture of an area covering latitudes $60^{\circ}-80^{\circ} \mathrm{N}$ and $10^{\circ}-30^{\circ} \mathrm{E}$ in the auroral region. Generally, the current estimates from satellite and ground are in good agreement. The results of this survey clearly show the average dependence of the auroral electrojet on season and local time. This is particularly true during periods of increased auroral activity. The correlation coefficient of the results is close to one in the region of sizeable ionospheric current densities. Also the ratio of the current densities, as determined from above and below the ionosphere, is close to unity. It is the first time that the method of Hall current estimate from a satellite has been validated quantitatively by ground-based observations. Among others, this result is of interest for magnetic main field modelling, since it demonstrates that ground-based observations can be used to predict electrojet signatures in satellite magnetic field scalar data.
\end{abstract}

Key words. Ionosphere (auroral Ionosphere; electric fields and currents; ionosphere-magnetosphere interactions)

Correspondence to: P. Ritter (pritter@gfz-potsdam.de)

\section{Introduction}

The most intense current system in the ionosphere is that of the auroral electrojets in the auroral oval. The strength and latitudinal position of these current flows depend on many factors, for example, on the solar zenith angle, solar wind activity and magnetospheric convection and substorm processes. The characteristics of the auroral electrojet have been of interest, since they reflect the dynamics and the processes at the magnetopause and in the outer magnetosphere. Traditionally, ground-based observations at auroral latitudes have been interpreted in terms of equivalent ionospheric currents. In case of additional information the different current components could be determined independently (e.g. Kamide and Richmond, 1982; Untiedt and Baumjohann, 1993, and references therein). A recent refinement of equivalent ionospheric current estimates from ground-based data has been presented by Amm and Viljanen (1999).

A detailed picture of the ionospheric current distribution can also be derived from satellite measurements, in particular from those in low Earth orbits. As an example, Zanetti et al. (1983) were the first to combine Magsat magnetic field and ground-based data from the IMS magnetometer array to determine the 3-D configuration of the ionospheric currents. The possibility of deriving horizontal ionospheric current estimates from the magnetic field scalar data was first demonstrated by Olsen (1996), who also employed Magsat data. In a later study, Moretto et al. (2002) applied this technique to data from other satellites.

In this paper we are presenting ionospheric currents which have been estimated independently from the ground and from satellite. The purpose of the study is (1) to present the statistical properties of the electrojet currents, (2) to cross-calibrate and verify these two interpretation techniques, and (3) to show how to use these methods to improve main field modelling results at high latitudes. As space data we used the highly accurate magnetic field measurements of the CHAMP satellite. The satellite was launched on 15 July 2000 and circles the Earth on a nearly circular orbit with an inclination of $87.25^{\circ}$. The orbit period is $\approx 93$ minutes. The satellite 


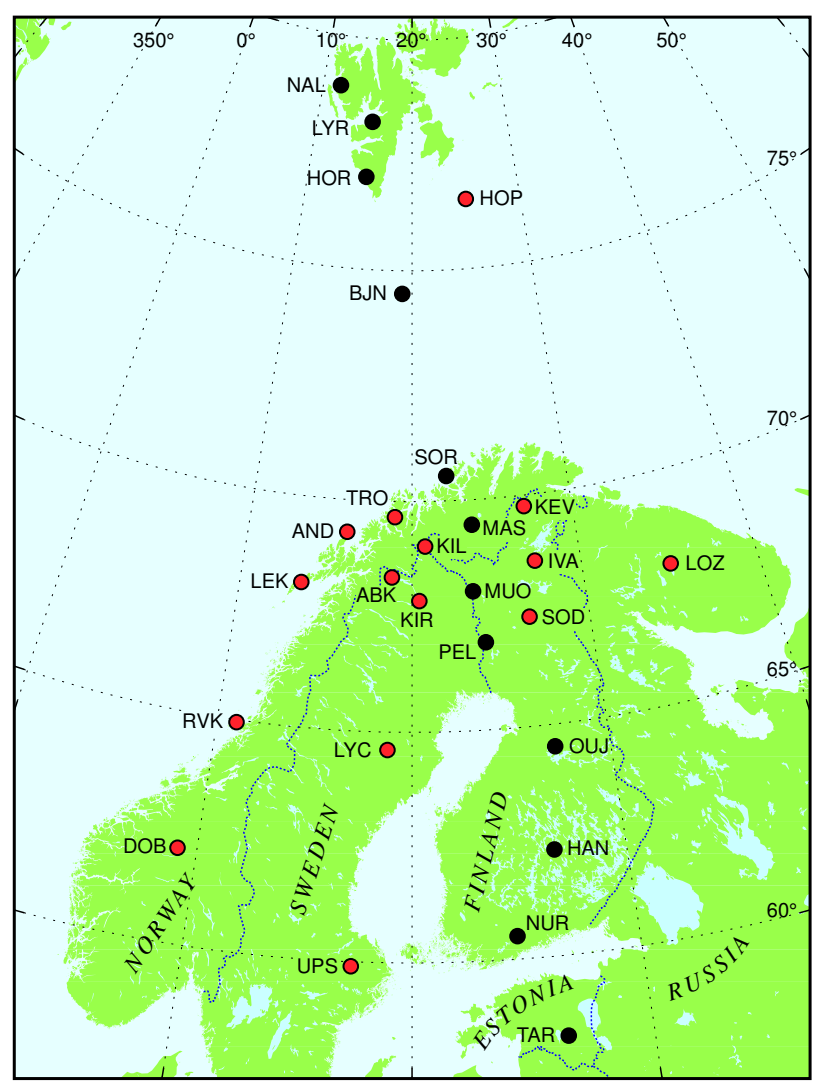

Fig. 1. IMAGE magnetometer network in 2002. Stations marked with black dots are used in the 1-D upward continuation.

performs more than 15 orbits each day. During the first two years the average height above the Earth's surface decreased from $455 \mathrm{~km}$ to $400 \mathrm{~km}$. This time window, August 2000May 2002, is chosen for a comparison of ground/satellite results. The drift of the orbit plane in local time (LT) is about $3 \mathrm{~h}$ per month. Consequently, the LT of the ascending node of the orbit moved through $24 \mathrm{~h}$ twice in those 22 months. As the period for repeat orbits is approximately 3 days during that time, the satellite passed the region chosen for the comparison with ground magnetometers at least twice in $72 \mathrm{~h}$, alternately on ascending and descending tracks.

The ground-based observations have been obtained from the IMAGE magnetometers. The IMAGE array (Lühr et al., 1998) consists of 27 magnetometer stations maintained by 10 institutes from Estonia, Finland, Germany, Norway, Poland, Russia and Sweden. The site locations are depicted in Fig. 1. For the most recent information on the programme the reader is recommended to visit the IMAGE website (http://www.geo.fmi.fi/image). Together with other groundbased recordings (by radars, riometers, all-sky cameras of the MIRACLE network) and satellite observations, IMAGE is an essential part in the investigations of high-latitude magnetospheric-ionospheric physics. IMAGE evolved in 1991 from the earlier EISCAT Magnetometer Cross, which was set up in 1982, and provides high-quality data useful for studies of auroral current systems, geomagnetic induction and long-term geomagnetic activity in the auroral region. The long profile covering geographic latitudes from 58 to 79 degrees is especially favourable for auroral electrojet studies. The complete array is used in this case to also compute the spatial variation and direction of the ionospheric current densities. The average auroral electrojets are observed mainly in a narrow region between approximately $65^{\circ}$ and $70^{\circ} \mathrm{N}$ of geomagnetic latitude. They are directed anti-sunwards, i.e. eastwards at dusk and westwards at dawn, in both hemispheres. The return currents cross the polar caps in an opposite direction, from the night to the dayside, and their lateral extension covers a larger region. Here, we focus on the auroral electrojet, because the station array covers the return current only marginally.

In this paper we briefly describe the methods used to determine the currents from space and from ground magnetic field data. Some typical examples show the general characteristics of the respective estimates. In Sect. 4 we present a statistical study of the electrojet characteristics and then compare the current estimates from CHAMP and the IMAGE array for a time period of 22 months. Some selected current density curves and contour maps of the results are shown. For a more quantitative comparison we perform a correlation analysis and compute the amplitude ratio of the obtained current densities independently for all latitudes and for specified local time intervals. Finally, we interpret the obtained results in the discussion section and compare them with earlier publications.

\section{Ionospheric Hall Current determined from CHAMP data}

The ionospheric currents at high latitudes comprise Hall, Pedersen and field-aligned currents (FACs). All three contribute to the magnetic fields measured by satellites. Groundbased observations respond primarily to the Hall currents at these latitudes. The magnetic effects of the FACs and Pedersen currents cancel each other below the E-region, if the ionospheric conductances are uniform (Fukushima, 1976). In order to distinguish between the different types of currents, we assume the Hall currents to close entirely in the ionosphere, whereas Pedersen currents are diverted into FACs. Under these conditions, and assuming vertical field-aligned currents, which is reasonable at high latitudes, only Hall currents contribute to the total field and can be determined from the scalar magnetic data of the Overhauser magnetometer on CHAMP. This topic will be addressed in more detail in the Discussion section.

For the estimation of the electric current density from single satellite magnetic field measurements the geometry of the current has to be known. We assume that in the polar regions the Hall currents can be approximated by a series of infinite line currents. This method has been developed and tested with Magsat and Ørsted data (Olsen, 1996; Moretto et al., 2002). For our computations the line currents are placed at 
a height of $110 \mathrm{~km}$ and they are separated by $1^{\circ}$ in latitude over an interval of $\pm 80^{\circ}$, centered at the closest approach of the satellite track to the geographic pole. Olsen's method (1996) additionally includes mirror currents to simulate induction effects; this issue is discussed further in the Discussion section. The magnetic field at orbital altitude caused by an eastward directed line current can be written as

$b_{x}=-\frac{\mu_{0} I}{2 \pi} \frac{h}{x^{2}+h^{2}}, \quad b_{z}=-\frac{\mu_{0} I}{2 \pi} \frac{x}{x^{2}+h^{2}}$,

where $b_{x}$ and $b_{z}$ are the northward and downward components of the generated magnetic field, respectively. $I$ is the current strength, $\mu_{0}$ is the susceptibility of free space, $h$ denotes the height above the current and $x$ is the northward displacement of the measurement point. The magnetic signature of the current in the field magnitude can be represented as

$\Delta F=|\mathbf{B}+\mathbf{b}|-|\mathbf{B}|$,

where $\mathbf{B}$ is the unperturbed ambient magnetic field in a corrected geomagnetic coordinate system. Since $\mathbf{b}$ is much smaller than $\mathbf{B}$, it is justified to replace Eq. (2) by the normalized dot-product between $\mathbf{B}$ and $\mathbf{b}$

$$
\Delta F=\frac{\mathbf{B} \cdot \mathbf{b}}{|\mathbf{B}|} .
$$

With this equation we obtain a linear relation between the total field deflection and the current strength, $I$. The intensity of each of the 160 line currents considered for the modelling can be derived from an inversion of the observed field residuals using a least-square-fitting approach.

For these calculations a static current system is assumed. Since CHAMP crosses the region at a speed of $4^{\circ}$ per minute, a certain smoothing occurs. At the orbital altitude, some $300 \mathrm{~km}$ above the atmosphere, we have a correlation length for a line current (here defined as the distance $x$ from the current centre to the location where $b_{z} / b_{x_{o}}=0.25$ ) of about $\pm 1000 \mathrm{~km}$. This corresponds to an averaging effect of approximately $5 \mathrm{~min}$.

For the inversion the magnetic data over a $160^{\circ}$ wide orbit segment are used. The scalar magnetic field readings are available at a rate of $1 \mathrm{~Hz}$, which is equivalent to 16 measurements per degree of latitude. In order to isolate the magnetic effect of the currents in the CHAMP measurements, the contributions from all other sources have to be subtracted from the scalar field readings. The main field is removed with the help of the recent CO2 model (Holme et al., 2003) employed up to degree and order 14. The lithospheric magnetisation is accounted for by subtracting the recent model by Maus et al. (2002). To eliminate the ring current effect, a $D_{S T}$ correction, according to Olsen (2002), is applied. Additionally, linear trends over the entire orbital arc are removed, to avoid effects due to asymmetric ring current distributions on the day and nightsides. To suppress any currents showing up spuriously at lower magnetic latitudes $\left(\theta_{m}<40^{\circ}\right)$, we applied a parabolic damping of the current density at the edges of the interval.
Figure 2 shows as an example of current estimates in the polar regions of the Northern and Southern Hemispheres over a period of 1 month. During that time, in October 2000, CHAMP was in a dawn/dusk orbit, thus crossing the auroral electrojets almost perpendicularly. The displayed time interval includes all 31 days of the month. The satellite tracks can be imagined as crossing the contour plots from top to bottom for each orbit. The auroral electrojets are prominent along two ribbons of enhanced current density at magnetic latitudes of about $\pm 70^{\circ}$. The positive amplitudes mark anti-sunward directed currents. The currents in the polar cap region are weaker and directed oppositely, from the night to the dayside, as expected. Strength and latitudinal extension of the current flow are correlated in general with the amplitudes of the $A E$ index.

In the subauroral regions (equatorwards of the auroral electrojets) the amplitude levels of the currents are small. At these latitudes a systematic difference for dawn and dusk is evident, as indicated by the light blue and yellow colors, respectively. This is not an edge effect of the model, since the considered orbit arcs are much longer than those shown in the contour plots and tapering is applied at the ends. The systematic difference in amplitude is rather a hint to a nonsufficient, standard $D_{S T}$ correction, which does not take into account the asymmetric ring current.

\section{Analysis methods for IMAGE data}

The simplest way to explain variations of the ground magnetic field is to assume that ionospheric currents flow in a fixed direction, for example, transverse to a magnetometer chain. Then it is possible to determine the ionospheric surface current density using the component of the field which is aligned with the chain. Typically, the auroral electrojet current flow is assumed to be in the east-west direction, and then the northward component of the field is used. We applied in this study the method based on the Fourier expansion to continue the field observed on the ground to the ionospheric level (Mersmann et al., 1979).

Two-dimensional equivalent currents can be determined with the method of spherical elementary current systems, as derived by Amm and Viljanen (1999), and validated in detail by Pulkkinen et al. (2003). In that case we obtain both the northward and eastward components of the surface current density at ionospheric level. Because IMAGE is quite a dense array, we could always use the 2-D method, but for reference we applied the 1-D approach to show that it is also often reasonable. The 1-D method is also of importance, if the same approach is to be carried out in an area where there is only a 1-D chain of stations available (for example, Greenland).

Induction effects due to the conducting Earth could be included by setting up an additional current system below the Earth's surface, but this is omitted here. We are mostly interested in disturbed events, and then the primary ionospheric contribution to horizontal magnetic variations close 

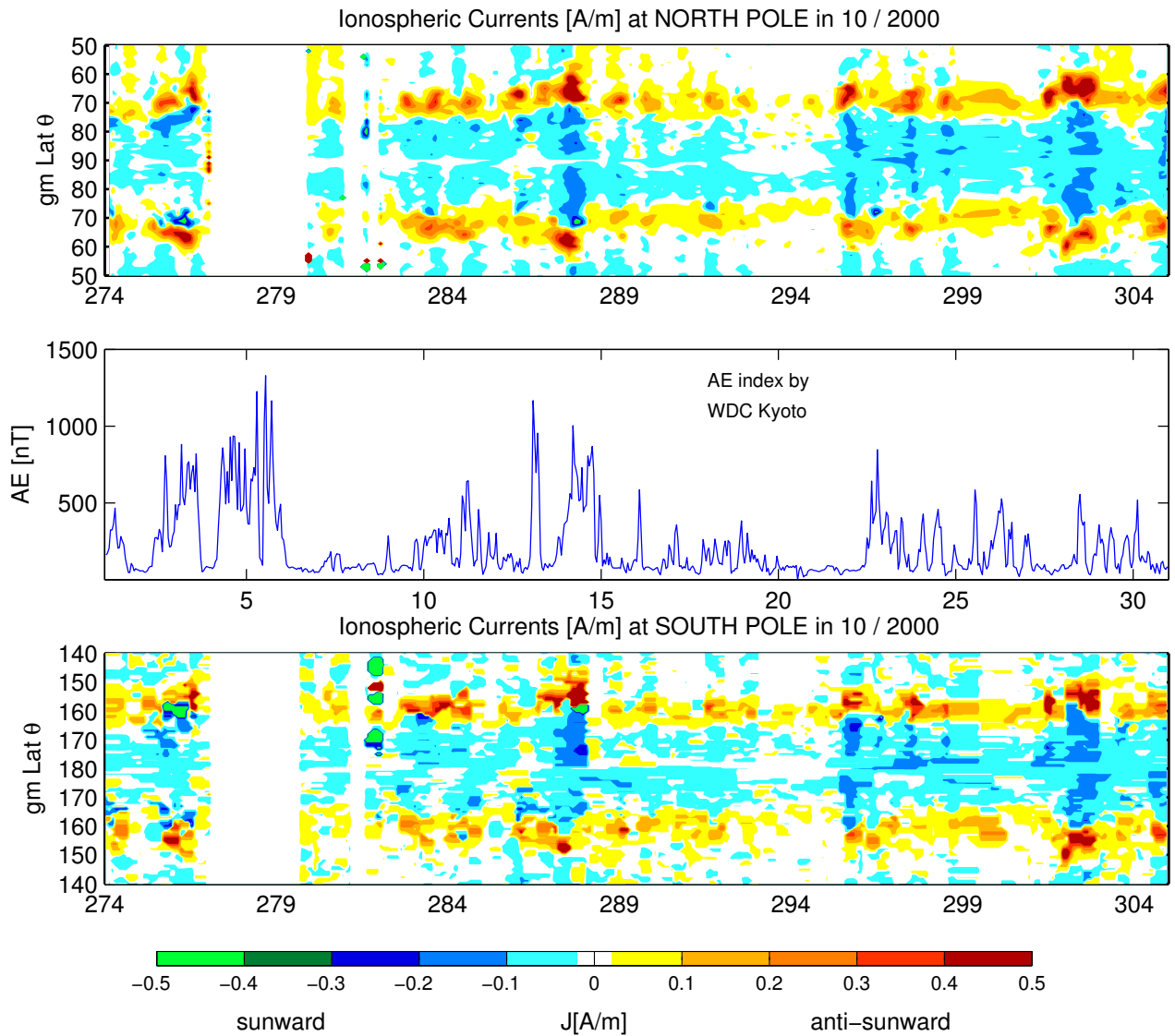

Fig. 2. Ionospheric currents in the polar regions of the Northern and Southern Hemispheres. For comparison the $A E$ index is plotted in the middle panel. The horizontal time scale spans one month, given as day numbers in MJD2000 format, i.e. the Modified Julian Days are counted from 01.01.2000, 0:00:00 UT.

to electrojet centres may be about $80 \%$ of the total variation (Tanskanen et al., 2001). Neglecting induction means that the ground-based observations provide slightly overestimated amplitudes of equivalent currents, but the geometric pattern of the current systems remains practically unchanged.

Before determining ionospheric currents from the groundbased observations, contributions from all other field sources have to be removed. For this study a particular effort was undertaken to derive reliable base values for all the stations. We used two criteria to select quiet days for the baseline determination. First, the global $K_{p}$ index must be equal or less than $1+$ during the last three hours of the day. Second, the local $A E$ index derived from IMAGE data during the last two hours of the day has to be less than $100 \mathrm{nT}$. There are 202 days fulfilling both conditions in the period January 2000 to May 2002.

After correcting some instrumental effects on magnetic readings during this period (e.g. recalibrations of the magnetometers or installations of new instruments), the secular variation correction is accounted for by the global satellite magnetic field model CO2 (Holme et al., 2003). No visible longterm trend $(<2 \mathrm{nT}$ per year) was left after that. Finally, a linear correlation between the $D_{s t}$ index and the field values seemed to exist, somewhat different for each station. A $D_{s t}$ correction thus derived was applied. The resulting baseline value $B_{\text {base }}$ at time $t$ is

$\boldsymbol{B}_{\text {base }}(t)=\boldsymbol{B}_{0}+\boldsymbol{C}_{S V} \cdot t+\boldsymbol{C}_{D_{s t}} \cdot D_{s t}+\boldsymbol{f}(t)$,

where $t$ is counted in days from 1 January 2000 (00:00 UT), $\boldsymbol{B}_{0}$ is the baseline value at $t=0, \boldsymbol{C}_{S V}$ is the secular variation coefficient taken from the $\mathrm{CO} 2$ model, and $\boldsymbol{C}_{D_{s t}}$ is the $D_{s t}$ coefficient. Due to intentional baseline changes or due to instrumental malfunctions, an additional, individual correction term $f(t)$ is necessary at some stations. A check was made excluding some stations with outliers not covered by $\boldsymbol{f}(t)$. A more detailed description of the baseline determination method and correction terms $\boldsymbol{f}(t)$ can be found in Sillanpää et al. (2003). A visual inspection of the field signatures showed that the automatic baseline selection is reasonable, if the magnetic effects caused by the currents are at least several tens of nT.

For 1-D equivalent current estimates, $B_{x}$ from the stations along the chain TAR-NAL in Fig. 1 can be used (TAR started to operate in September 2001, and it was not considered here). Equivalent current densities were given at the height of $100 \mathrm{~km}$ along a geographic meridian from about $60^{\circ}$ to $79^{\circ}$ 

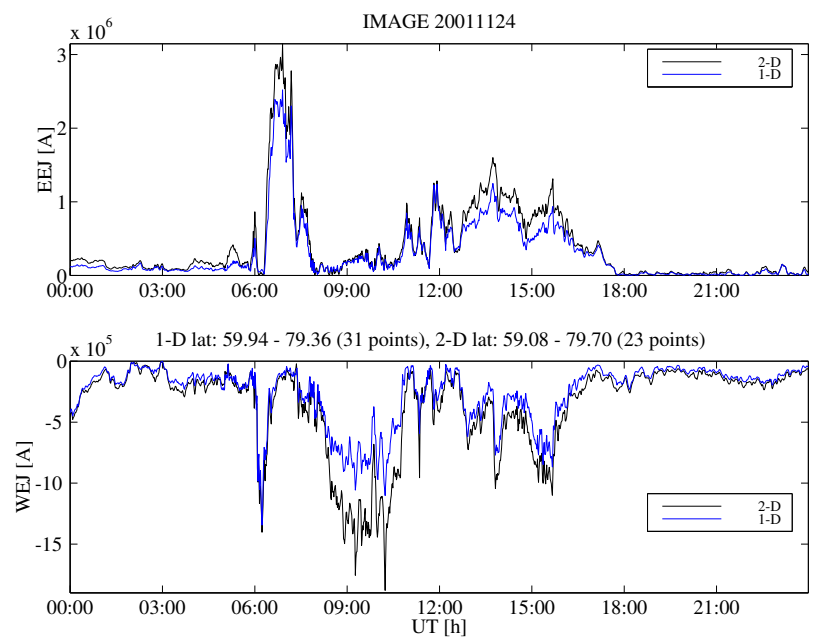

Fig. 3. Total eastward and westward electrojet equivalent ionospheric currents over IMAGE on 24 November 2001. The blue curve corresponds to the 1-D upward continuation, whereas the black curve shows the result by the 2-D method of spherical elementary current systems.

geographic latitude with a spacing of about $0.7^{\circ}$. This meridian is not precisely aligned with the magnetometer chain, but it can be imagined to cross the central parts of IMAGE. Due to the 1-D assumption, the zonal location of the meridian does not matter.

For the 2-D equivalent current estimates, we used a set of about 1600 elementary current systems at a height of $100 \mathrm{~km}$ in a regular grid $\left(54^{\circ}-84^{\circ} \mathrm{N}, 2.5^{\circ}-42.5^{\circ} \mathrm{E}\right)$ covering the whole IMAGE array with a sufficient outward extension. Amplitudes of the elements were determined by using the horizontal components $B_{x}$ and $B_{y}$ of all available IMAGE sites. We considered CHAMP footpoints in the area of $60^{\circ}-78^{\circ} \mathrm{N}$ and $10^{\circ}-30^{\circ} \mathrm{E}$, where edge effects do not cause artifacts.

Although at certain times 1-D and 2-D equivalent currents may be quite different, the overall agreement is reasonably good, as shown in Fig. 3. The total amplitudes of the electrojets are underestimated by the 1-D method. This can be understood when considering the horizontal magnetic field of a line current of finite length with vertical currents at both ends. Assume for simplicity that the observation point is just below the centre of the horizontal segment. Keeping the amplitude of the current fixed, but increasing the length of the horizontal part, the horizontal field increases, as can be easily deduced by applying the Biot-Savart law. Consequently, the 1-D method underestimates the amplitudes, because real currents are never infinitely long. However, at times, the electrojet seems to be very long, as around 06:00 UT for the WEJ case in Fig. 3, where both methods yield very much the same total current.

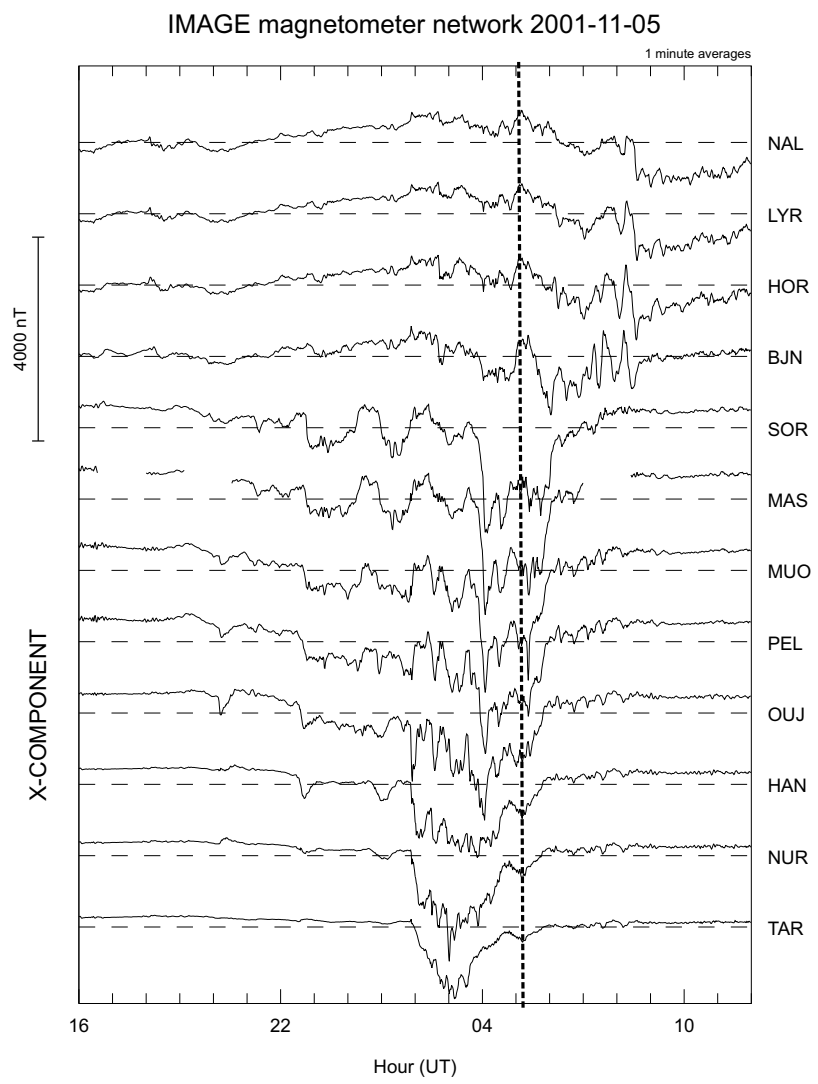

Fig. 4. Magnetograms of the IMAGE stations (for locations refer to Fig. 1) of the storm event starting on 5 November $2001 ; B_{x}$ component.

\section{Comparison of satellite and ground-based measure- ments}

After having presented the techniques for estimating ionospheric Hall currents, both from space and from ground, we will apply them for auroral current studies.

\subsection{An event study}

We are going to inspect an active period. As an example we selected the strong magnetic storm of 5/6 November 2001. It reached $D_{S T}$ values of $-277 \mathrm{nT}$. The storm commences, as can be seen in Fig. 4, shortly after 20:00 UT on 5 November. The event start is also evident from the decrease of $D_{S T}$. The actual main phase, however, starts only on the next morning, accompanied by a large SSC at 01:53 UT. The electrojet centre moves as far south as 58 around 03:00 UT. During the subsequent hours, the auroral electrojet retracts poleward, reaching $68^{\circ}$ at 05:00 UT. The activity stays high until 06:00 UT, reaching amplitudes of up to $2400 \mathrm{nT}$ and decays only after that time.

At about 05:07 UT CHAMP passed the IMAGE array from north to south (dashed vertical line in Fig. 4). At that time the magnetic field is still strongly deflected. Figure 5 shows the equivalent current distribution as derived by the 


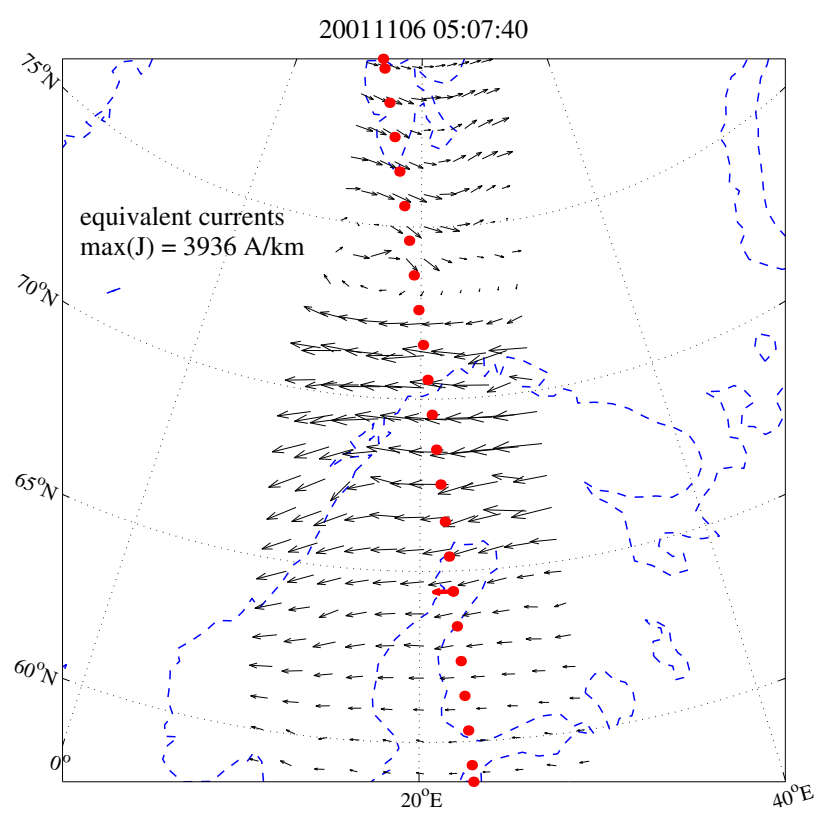

Fig. 5. Snapshot of equivalent currents on 6 November 2001, determined by IMAGE (black arrows) and CHAMP footprints (red dots).

2-D method for the time of the CHAMP overflight. There are very strong westward currents, reaching almost $4 \mathrm{~A} / \mathrm{m}$ in northern Scandinavia. Poleward of Bear Island we find weaker eastward currents. A direct comparison between the ground and satellite estimates is presented in Fig. 6. The blue curve represents current densities derived from ground-based data and the red currents denote those from satellite readings. Both curves track each other quite closely. Ground-based estimates are slightly higher, on average by $15 \%$.

In the same diagram we also show the field-aligned currents (FACs) estimated from CHAMP magnetic field vector data. For this coarse comparison we have assumed infinite FAC-sheets perpendicular to the flight direction. Poleward of $74^{\circ} \mathrm{N}$ there are no significant FACs. Further south we find a distinct downward current region. When going equatorward a series of up- and downward FAC sheets follows where the upward flow gradually starts to dominate.

By combining all these measurements we obtain a rather consistent picture of the morning side polar convection cell. The downward FACs at $72^{\circ} \mathrm{N}$ can be associated with the Region 1 currents. Poleward we find the weaker polar cap eastward (sunward) currents. Equatorward there is the very intense westward auroral electrojet. The transition from the Region 1 to the Region 2 occurs above the auroral electrojet and is characterized by alternating upward and downward FACs. This signature suggests that the plasma convection in the magnetosphere is not entirely laminar but may form vortices at the interface between tailward and sunward flows. Consequently, we find the most intense upward FACs not in the Region 2 proper but above the auroral electrojet, giving probably rise to a high conductivity.
The size of the polar cap is not particularly large during the inspected passage. The boundary marked by the convection reversal in Fig. 5 occurs at about $70^{\circ}$ magnetic latitude (at the IMAGE region corrected magnetic latitudes are about $3^{\circ}$ lower than geographic ones). At the time of the main phase of the storm (03:00 UT) the boundary is $10^{\circ}$ further south. This implies a polar cap with a radius of $30^{\circ}$. The resulting magnetic flux stored in the tail during that time is, therefore, about 4 times higher than normal. The release of this magnetic energy causes the very intense currents.

\subsection{A statistical study}

During a period of 22 months, we identified 490 satellite overflights across the region of the IMAGE magnetometer array. The ionospheric currents were determined from CHAMP data and independently computed from the groundbased measurements using the 2-D method, as described above. The full set of stations supplied the database for the 2-D approach. Figure 7 shows the current density profiles of 16 overflights as examples. The agreement of the curves is good in general, indicating that the signatures of the ionospheric currents in the magnetic field can be detected equally clear on the ground and at satellite heights.

Current density profiles for all overflight events during the time period MJD 215-MJD 880 (Modified Julian Days) are shown in Fig. 8, sorted by ascending and descending tracks on the left and right side, respectively. Therefore, the magnetic local times scales (MLT) of the left and right frames differ by $12 \mathrm{~h}$, and accordingly, the derived currents have predominantly opposite signs. Positive currents (yellow and red) are directed eastward, whereas negative (blue and green) currents flow westward.

Currents determined from CHAMP magnetic field data are displayed at the top, and those from the IMAGE stations are presented in the bottom frames. During periods of increased activity, the current estimates are generally in good agreement, and the coincidence of the prominent amplitude maxima is obvious. The light-blue band (with $J \approx-0.1 \mathrm{~A} / \mathrm{m}$ ), visible around $65^{\circ}$ in the satellite data, is presumably due to short-scale crustal magnetic features in the Kiruna area, which are not resolved and removed completely by the lithospheric model used for our crustal correction.

The latitudinal displacement of the mean current locations with local time is clearly visible: around noon they are retracted poleward by several degrees, whereas shortly before midnight they reach their most equatorward position.

Besides the local time dependencies there are marked seasonal effects. During the winter solstice, the average activity is strongly reduced. This fact is particularly evident in the morning up to pre-noon hours. In our survey this time sector was sampled during winter months on days around MJD 760 by ascending tracks and around MJD 380 by descending tracks. For a period of almost 90 days the current densities hardly exceed the noise level. We find around midnight in winter individual storm/substorm events (MJD 350 
Hall Currents and FACs above IMAGE Region, dsc.

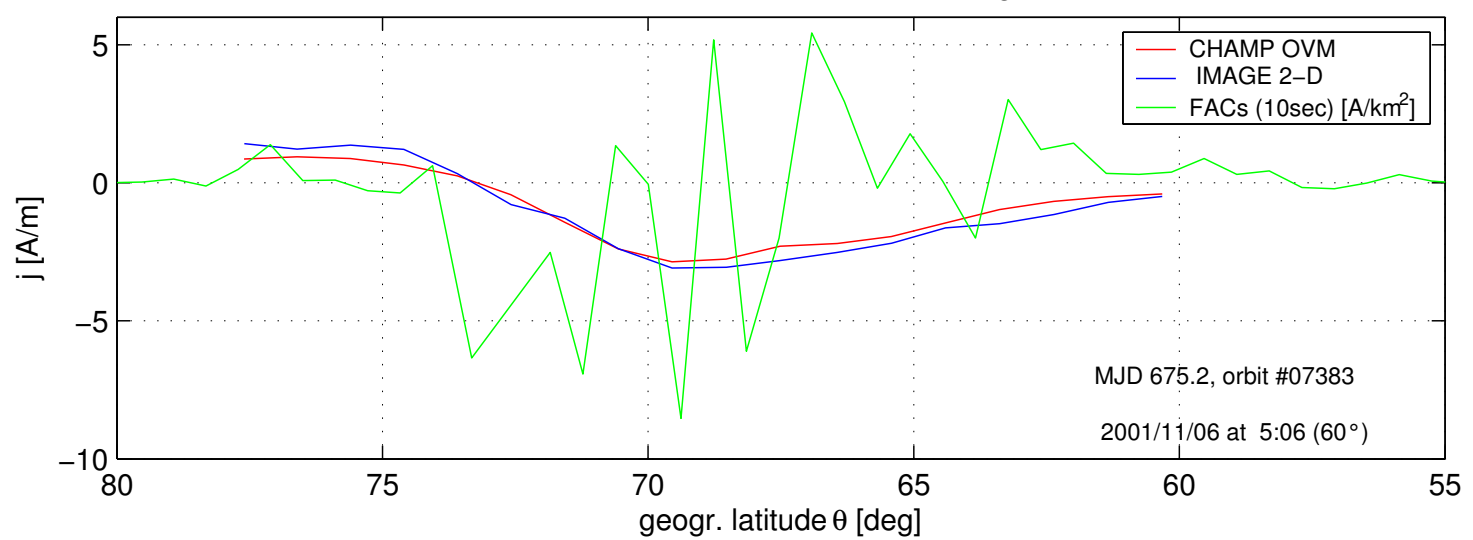

Fig. 6. Hall currents and field-aligned currents (10-s running mean) during the storm event on 5/6 Nov. 2001.

Hall Currents above IMAGE Region, asc.
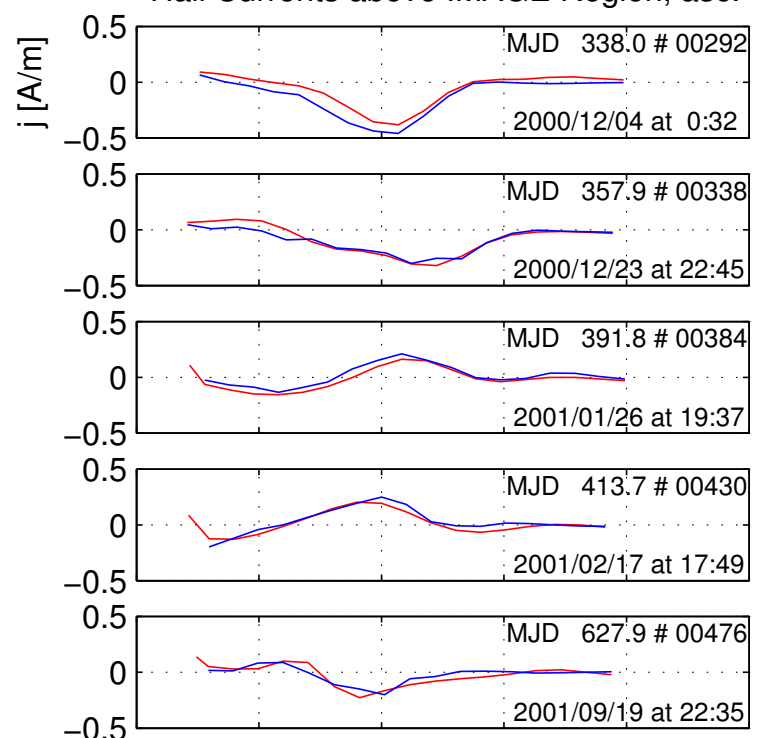

$0.5 \quad$ 'MJD 635.9 \# 00491
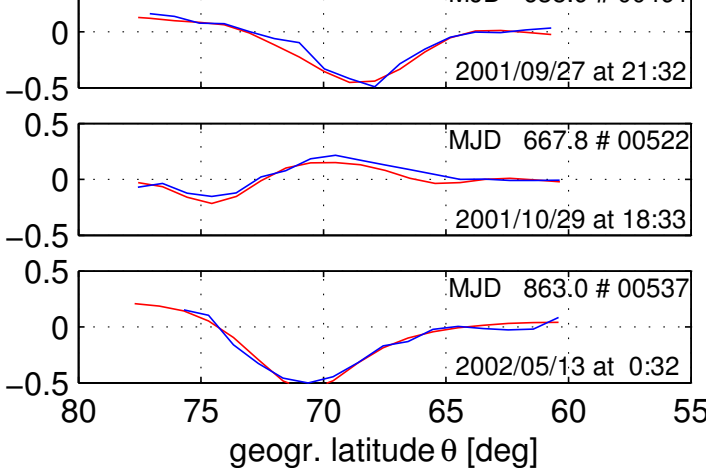

Hall Currents above IMAGE Region, dsc.
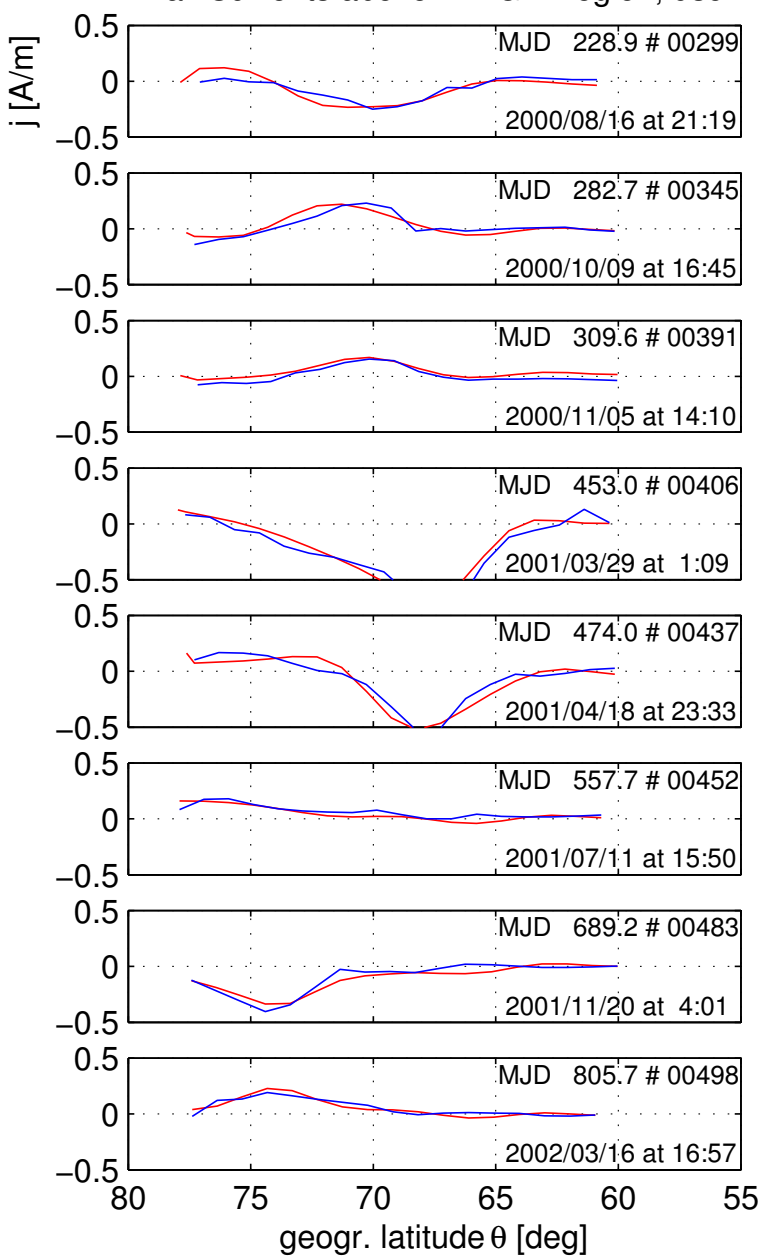

Fig. 7. Comparison of the current densities derived from CHAMP (red lines) and IMAGE ground-based measurements (blue lines) between Aug 2000 (MJD 215) - May 2002(MJD 880) on N/S profiles across the IMAGE array $\left(60^{\circ}-80^{\circ} \mathrm{N}\right.$ geographic latitudes). Left side: the satellite crossed the region on ascending tracks; right side: on descending tracks. MJD = Modified Julian Days (format see Fig. 2). 

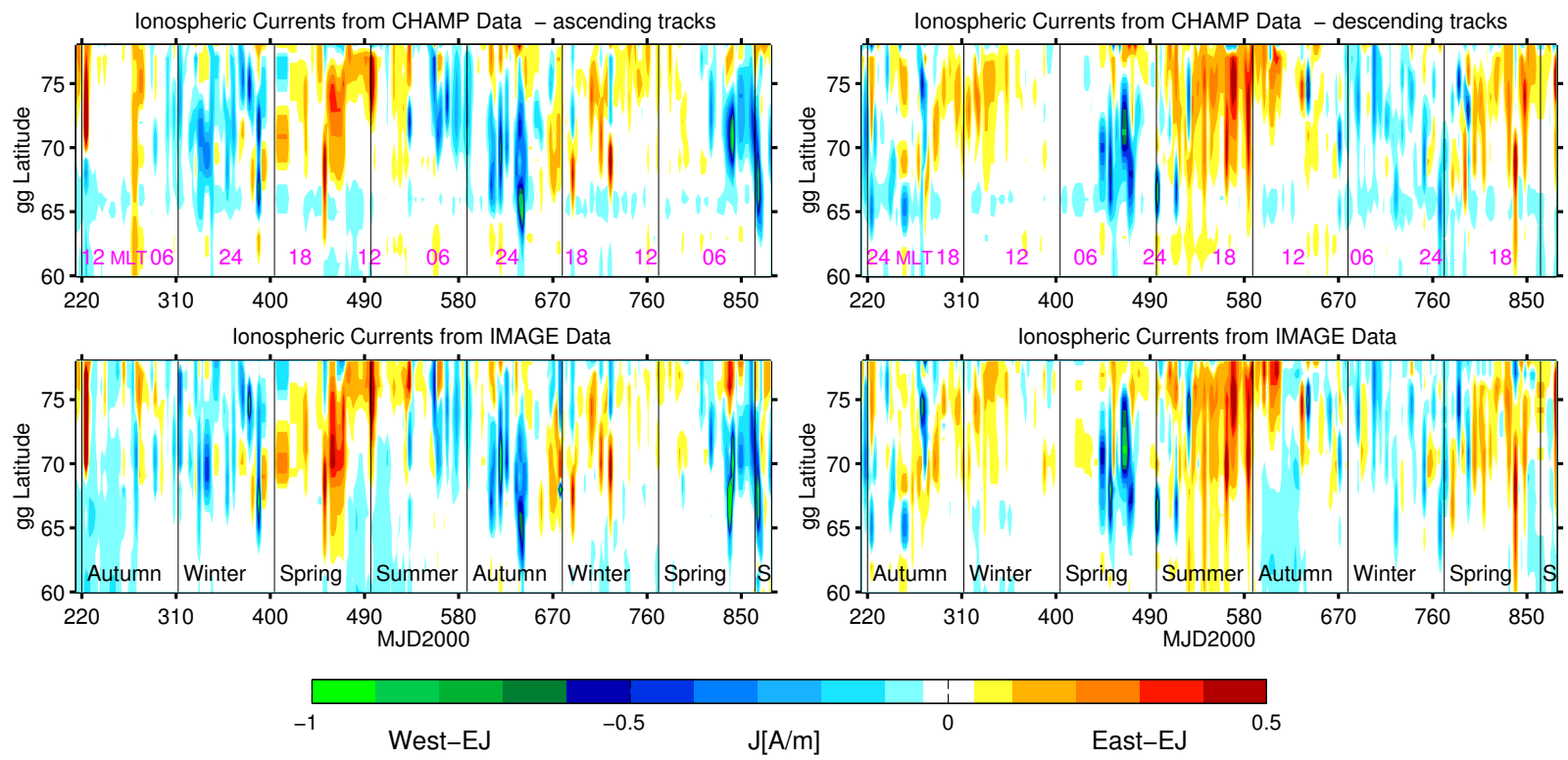

Fig. 8. Ionospheric currents for all 490 overflights during the time period MJD 215-MJD 880. Upper frames: determined from CHAMP data above the IMAGE region; lower frames: determined from IMAGE data. Left side: only ascending tracks; right side: only descending tracks. The red numbers in the top frames give the respective magnetic local times (MLT).
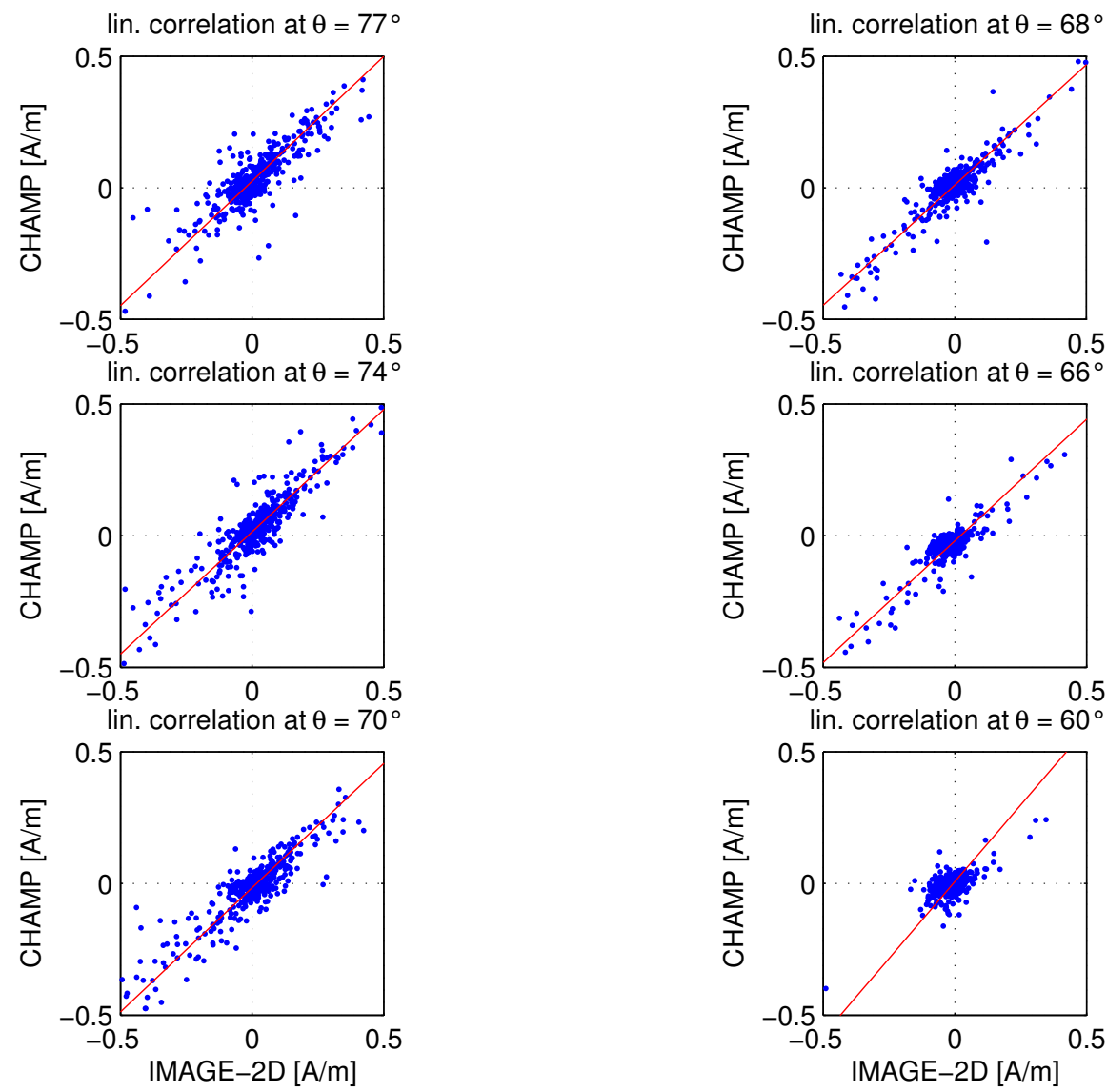

Fig. 9. Scatter plots of the ratios of current densities at selected latitudes determined from IMAGE and CHAMP. Linear regression lines are added. 
ascending and MJD 730 descending), but during the time inbetween there is generally low activity.

According to Russel and McPherron (1973), equinox seasons are characterized by enhanced magnetic activity. From our observations we can confirm an increase in substorm-related westward auroral electrojets during these seasons, when they are sampled around midnight hours (asc.: MJD 620, 860; desc.: MJD 470). When sampled at daytime, the Russel-McPherron effect is less pronounced. Only in one out of four possible cases did we find enhanced eastward currents (asc.: MJD 450). During the summer solstice, the eastward currents in the afternoon sector are quite prominent (desc.: MJD 560). This is probably due to greatly enhanced ionospheric conductivity caused by the continuous solar illumination during this season.

Our systematic survey samples all seasons independent of activity and thus provides an unbiased view of the auroral electrojet current distribution.

\subsection{Correlation analysis of ground/satellite results}

A more quantitative measure for the agreement of the results derived from space and ground data can be obtained, if the properties and characteristics of the amplitude ratios of all available overflights are analysed. For that purpose Fig. 9 presents scatter plots of the CHAMP versus IMAGE current densities at selected latitudes along the IMAGE meridional profile. The solid lines mark the linear regressions computed for the case where the more homogeneous satellite results ( $y$-axes) are assumed to be free of errors.

Only a few data points scatter completely away from the regression lines. The distribution of the ratios is clearly linear only down to $\approx 63^{\circ} \mathrm{N}$ of geographic latitude. For lower latitudes the current intensity becomes very small due to the rapidly decreasing conductivity.

Figure 10 pictures the results of the correlation analysis determined for each degree of latitude individually. From top to bottom the diagrams show the correlation coefficients $R_{c}$, average current densities, and the regression coefficients of all scatter plots, namely the amplitude ratios between IMAGE and CHAMP (inverse regression line slope) and local bias (regression line offset). These quantities were computed from the current estimates obtained over the IMAGE array in the region $\theta=60^{\circ} \ldots 80^{\circ} \mathrm{N}$.

The second frame shows the average magnitude of the current densities along the profile, individually for satellite and ground-based data. The estimated current densities are fairly low, on average, indicating that most of the overflights occurred during quiet periods. Starting from polar latitudes, the intensity obtained from CHAMP rises, attaining a plateau between $\theta=74^{\circ}$ and $69^{\circ} \mathrm{N}$. Thereafter it drops off. Current densities estimated from IMAGE data are generally higher. They reach a first maximum at $\theta=74^{\circ} \mathrm{N}$. A second maximum is attained at $68^{\circ}-70^{\circ} \mathrm{N}$. The local minimum in-between is co-located with the station gap between SOR and BJN (cf. Fig. 1). For $\theta<68^{\circ} \mathrm{N}$ the two curves fall off gradually, reaching the noise level at $63^{\circ} \mathrm{N}$. The 2-D
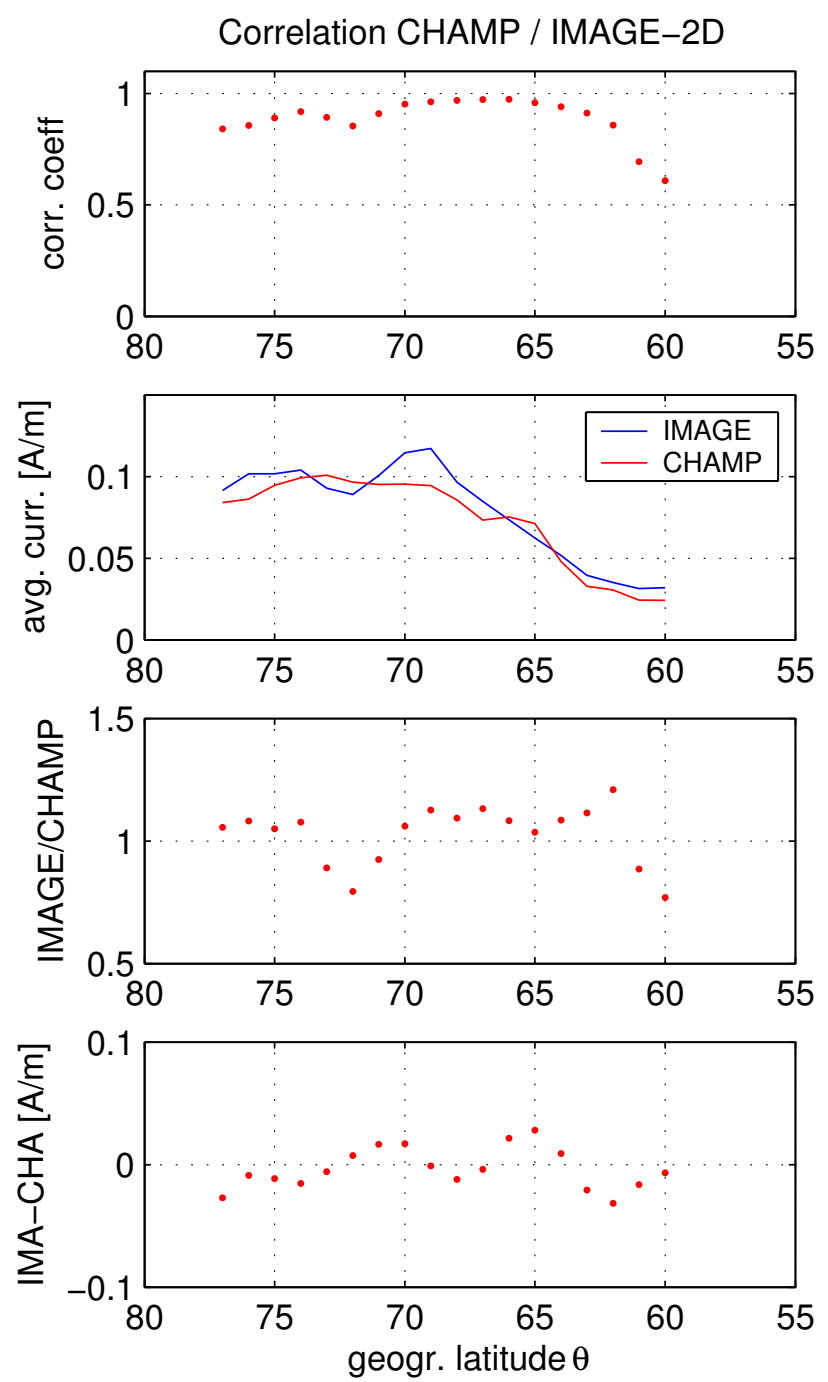

Fig. 10. Correlation analysis of the CHAMP versus IMAGE current estimates. From top to bottom: correlation coefficient $R_{C}$, average current density, regression coefficients, i.e. amplitude ratio (inverse regression line slope) and regression bias (regression line intercept).

IMAGE results yield values that are very much the same as those obtained from the satellite measurements.

The correlation coefficient $R_{c}$ in the top graph is close to one. Between $\theta=65^{\circ}$ and $70^{\circ} \mathrm{N}$ we obtain $R_{c}=$ $0.96 \pm 0.014$. A shallow minimum occurs where we have poor ground data support over the sea. Naturally, the correlation drops off in the region south of the electrojet. The third graph shows a similar picture, the amplitude ratio IMAGE to CHAMP stays close to one in the region of high current density. The average value for the ratio between $\theta=63^{\circ} \ldots$ $70^{\circ} \mathrm{N}$ is $1.08 \pm 0.01$. The offset of the regression lines (intercept), as shown in the bottom panel, may be caused either by residual crustal features in the CHAMP data or by remaining imperfections in IMAGE station baselines. The residuals between the linear regression and the data points (not shown) have an rms-value of about $40 \mathrm{~mA} / \mathrm{m}$. This may be regarded as the current detection limit. 
After having analysed the complete data set in one run, we will check now whether the agreement between ground and satellite estimates is dependent on the local time sector. For this purpose the data are divided into four parts, ordered by magnetic local time MLT (at the IMAGE region MLT is about $2.5 \mathrm{~h}$ ahead of UT). The same correlation procedure as before was applied to all the subsets. Figure 11 shows the results individually for the time sectors: 02:00-08:00 MLT, 08:00-14:00 MLT, 14:00-20:00 MLT and 20:00-02:00 MLT. These sectors are chosen to be symmetrical to the auroral convection pattern which is slightly skewed towards earlier times.

The smallest amplitudes are encountered around noon, as expected. Increasing amplitudes are recorded towards the evening and throughout the night. They finally reach a peak in the morning sector. This trend is also reflected in the curve of the correlation coefficient. At noon we find $R_{c}>0.8$ only north of the Scandinavian mainland, while the same condition is met almost over the entire array during early morning hours. The obtained ratio between IMAGE and CHAMP current intensities is obviously only reliable in cases of good correlation. If we limit our attention to results with $R_{c}>0.9$, we obtain the ratio IMAGE over CHAMP $\left(Q_{i}\right)$ for the individual MLT sectors: in the morning $Q_{i}=1.15$; in the evening $Q_{i}=1.12$, and at midnight $Q_{i}=1.08$. The consistently larger estimates from ground-based observations are probably caused by the induction effect, which will be addressed in more detail in the Discussion section. Finally, we might state that, regardless of local time, the agreement between the two independent methods of current estimates is good, as long as the intensity is above the noise level of $40 \mathrm{~mA} / \mathrm{m}$.

\section{Discussion}

We have compared estimates of ionospheric currents derived from satellite total field measurements with those obtained from ground-based observations of the horizontal magnetic field components. For this study all available 490 CHAMP passes over the IMAGE array during the considered 22 months were taken into account. There has been no selection of data according to the activity level. We wanted to study the features of the ionospheric Hall currents under all conditions.

An important prerequisite for a reliable current estimate is the proper separation of the magnetic signature generated by the ionospheric currents from all the other field contributions. We have put particular emphasis in this task. A dedicated study was conducted by Sillanpää et al. (2003), to calculate the true baselines for all 27 magnetometer stations of the IMAGE array. As mentioned in Sect. 3, the ground observations have been corrected additionally for the secular variation and the ring current $\left(D_{S T}\right)$ effect. In the same manner, we employed the most recent field models for the correction of the CHAMP data: the CO2 model (Holme et al., 2003) for the main field, which includes the secular variation and $D_{S T}$ corrections. The shorter wave length crustal anomalies were accounted for by the model of Maus et al. (2002). It is to our knowledge the first time that, for a statistical ionospheric current study, all of these corrections have been applied. This makes our results unique and important.

The two current estimates have been derived completely independently by the two groups involved, without any parameter adjustments or scale fitting between the satellite and ground-based data processing. Even the current models employed are quite different. The high degree of correlation between the two sets of results $\left(R c=0.96\right.$ between $65^{\circ}$ and $70^{\circ} \mathrm{N}$ ) is thus a very convincing indication that both techniques yield reliable results.

An important question in this context is, do both systems measure the same ionospheric currents? From the Fukushima theorem (Fukushima, 1976) we know that, under the assumption of vertical field-aligned currents (FAC) and a homogeneous ionospheric conductivity, the magnetic effect of Pedersen currents cancel on the ground. Above the ionosphere FACs and Pedersen currents do not cancel, but both systems generate exclusively horizontal magnetic fields. With the assumed field geometry these magnetic fields are perpendicular to the ambient field and thus cause only negligible contributions to the field magnitude. If the inclination of the ambient field deviates slightly from vertical (e.g. incl. $\approx 80^{\circ}$ at auroral latitudes), some magnetic effects from the FAC-Pedersen current circuit will leak to the ground, and above the ionosphere we will find a small field-aligned contribution from this circuit. However, in the case of elongated field-aligned current sheets, which is quite common in auroral regions, the FAC and Pedersen currents, even for oblique geometry, will vitually cancel again on the ground (apart from the edges), and above the ionosphere only perpendicular magnetic fields are generated.

From all these considerations we may conclude that the high-latitude FAC-Pedersen current circuit has only a minor effect on ground-based observations and equally little on the field magnitude above the ionosphere. What we are interpreting with both systems are thus primarily ionospheric Hall currents. If we allow for conductivity gradients, which are quite common at auroral latitudes, a coupling of the Hall and Pedersen current occurs (Glassmeier, 1984). Therefore, a more general statement would be that both facilities observe the source-free current component. For the sake of simplicity we will, however, stick to the term Hall current as an equivalent to the source-free component. For one storm event we have presented the details of the observations both from ground and satellite. Combining the derived Hall currents with the FACs of these dawn sector observations provides a comprehensive picture of the 3-D current configuration. It is most obvious that we do not find the classical transition from Region 1 to Region 2, as suggested by Iijima and Potemra (1976), but rather observe an alternating series of upward and downward directed FACs. The mean value of neighbouring FAC sheets gradually changes from downward to upward. Therefore, the classical R1/R2 transition describes just the average condition. The fine structure of 
Correlation CHAMP/IMAGE, MLT: $02-08 \mathrm{~h}$
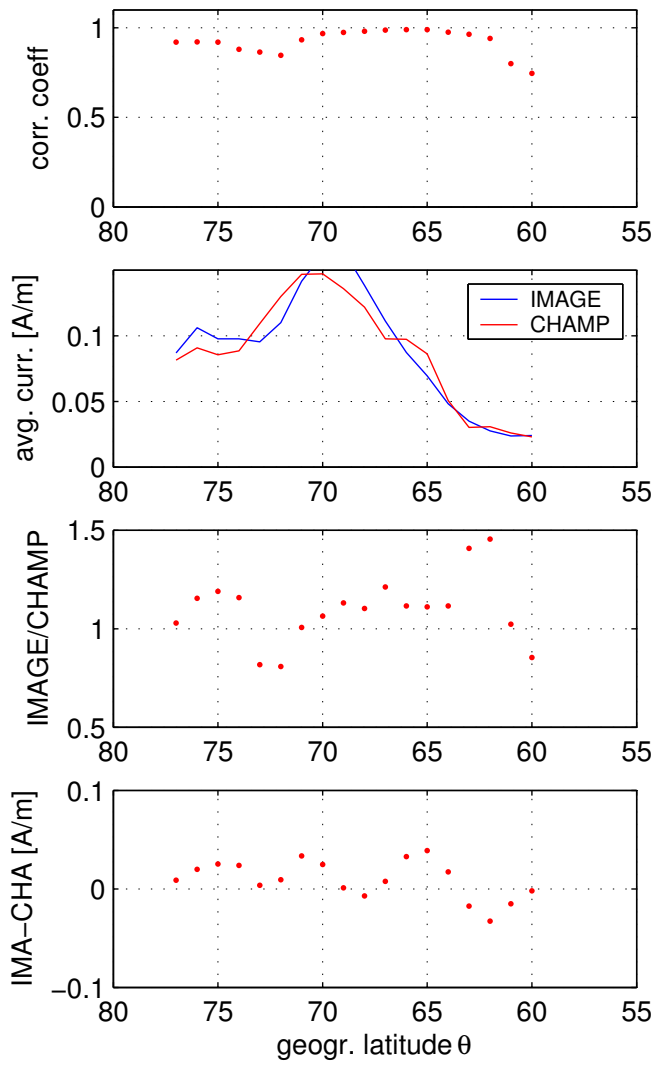

Correlation CHAMP/IMAGE, MLT: $14-20 \mathrm{~h}$
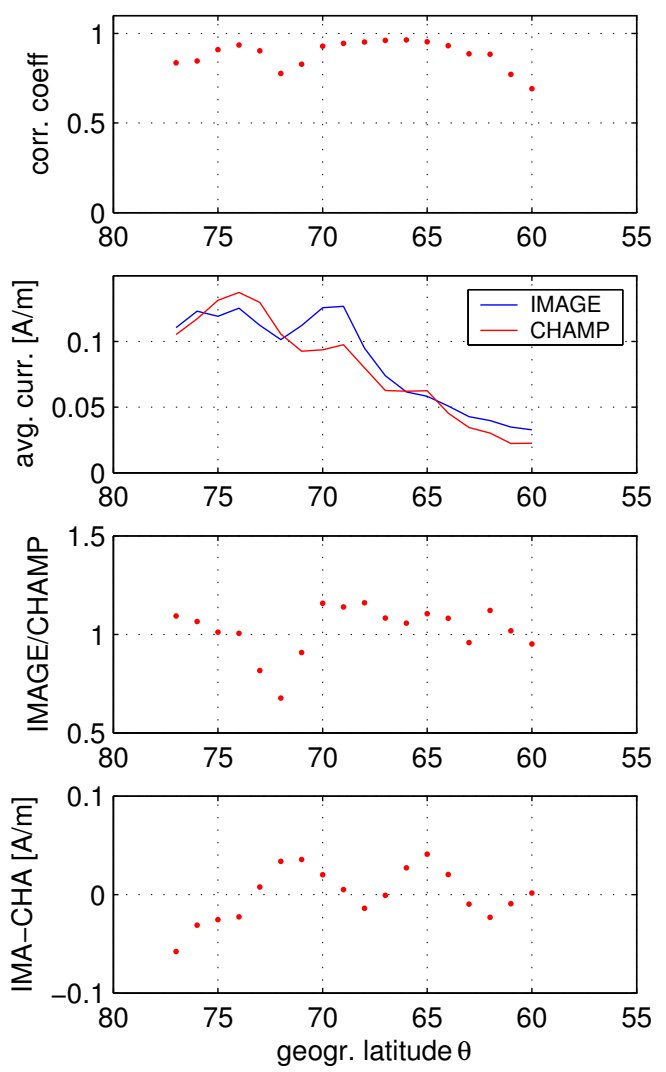

Correlation CHAMP/IMAGE, MLT: 08 - $14 \mathrm{~h}$
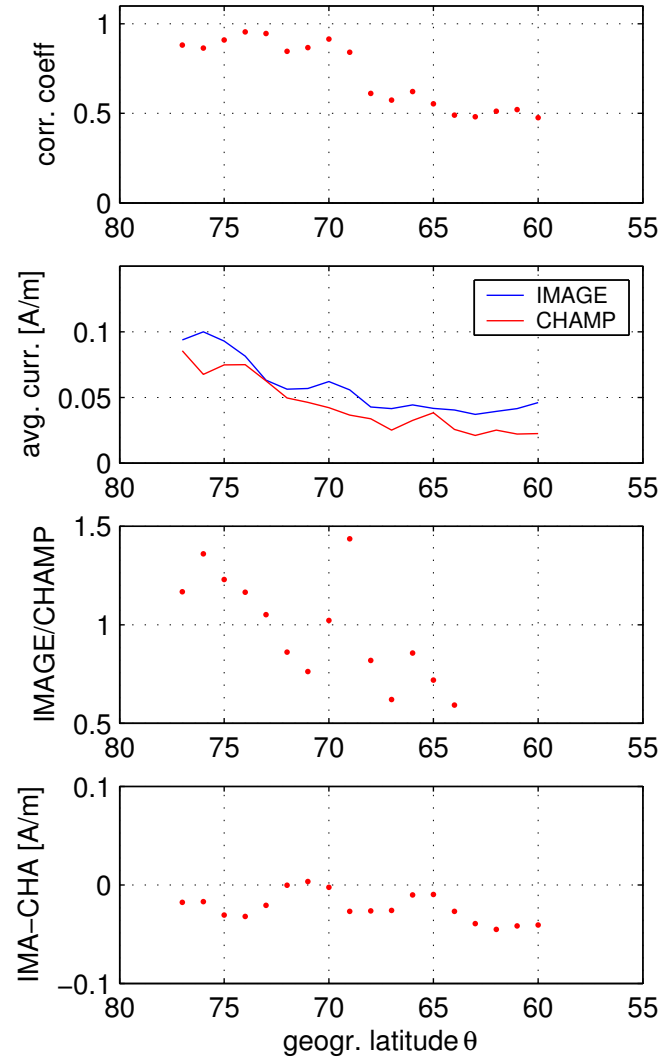

Correlation CHAMP/IMAGE, MLT: $20-02 \mathrm{~h}$
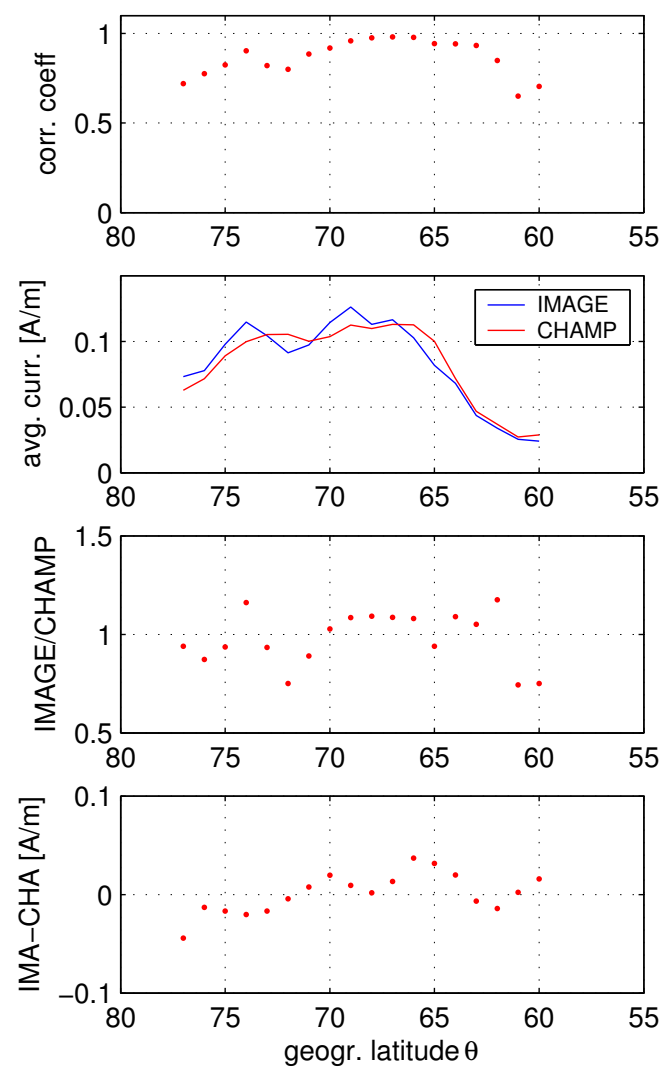

Fig. 11. Correlation analysis of the CHAMP versus IMAGE current, the estimates are grouped in 4 magnetic local time sectors. 
FAC sheets is of concern for understanding things such as the conductivity distribution or the Joule heating. The characteristics and fine structure of field-aligned currents, however, are not pursued any further in this paper.

In our statistical study we presented the systematic day-today variability of the Hall current distribution in the auroral zone. In Fig. 8, showing the activity over the whole considered period, seasonal dependencies and local time effects are clearly visible. The most important relation seems to be the solar zenith angle dependency. In summer the overall activity is higher, in winter the background activity is low. Individual storms/substorms clearly stand out from the background activity. During these events, precipitating particles enhance the otherwise low conductivity locally. In the pre-noon to noon sector there is often a long void of activity during the winter season. During equinox seasons, an enhancement in magnetic activity is expected according to Russell and McPherron (1973). We find that this effect is confined primarily to the nightside where a higher occurrence frequency for substorms is observed. Around noon no significant influence can be reported. Here, the solar zenith angle seems to be the dominating controlling factor for ionospheric current activity. Obviously, precipitating particles have only a minor effect compared to solar irradiation on the conductivity in this local time sector. Most previous statistical studies on auroral activity were event-oriented or looked at the auroral oval on the whole, for example, employing the $A E$ index. Here we look at the seasonal and local time effects at the same time. This study leads to interesting conclusions, for example, concerning the conditions to find periods of extra low activity suitable for magnetic main field modelling.

An important purpose of this paper is the cross-calibration and verification of current estimates from the ground and satellite. The correlation between current estimates from these two facilities is generally very high, as shown in Fig. 10. In the region of good station coverage and reasonable electrojet activity, $\theta=65^{\circ} \ldots 70^{\circ} \mathrm{N}$, the correlation coefficient ranges around 0.96 . Here, the standard deviation from the linear regression line amounts to only $40 \mathrm{~mA} / \mathrm{m}$. Equatorward of the auroral electrojet, where the average current density decreases and approaches the value of the standard deviation, the correlation falls off rapidly. The current densities derived from ground-based data are generally larger than those from the satellite (cf. Fig. 10). An exception is observed at latitudes around $72^{\circ} \mathrm{N}$. The SECS method tends to underestimates the current intensity in an area of insufficient data coverage. This is the case in the gap between the Scandinavian mainland and Bear Island. We have excluded this latitude range when considering the IMAGE/CHAMP amplitude ratio. For the linear regression we obtain a more consistent latitude profile when assuming the CHAMP-related currents rather than those from ground estimates to be free of errors. The smoother results may be explained by the more homogeneous satellite data set, where all readings come from the same instrument and the sampling is continuous and equally spaced.
Interesting results emerged from ground/satellite correlations that were grouped by local time sectors. In the noon sector, with its very few active events, the correlation is rather poor, therefore those results are not given any further consideration. Within the other sectors, however, significant correlations are obtained at least over a part of the IMAGE array. We used the parts with a correlation coefficient above 0.9 to determine the IMAGE to CHAMP amplitude ratio. Within a given time sector the considered ratios have mainly the same value (cf. Fig. 11). The smallest value (1.08) is encountered around midnight, the largest (1.15) in the morning sector. We regard the higher amplitude from ground-based data as due to induction effects. The slight dependency on local time of this ratio may well be caused by the different current geometries. In the morning sector high correlation is obtained down to the south of Finland. At the latitudes $62^{\circ}$ to $63^{\circ} \mathrm{N}$ we find significant ratios of 1.4.

From theoretical considerations it is expected that estimates from the satellite fall short compared to those from ground. The magnetic effect of currents induced in the ground will add to the ground-based measurements but reduce the signal above the ionosphere (Olsen, 1996). For the satellite estimates we use the scalar magnetic field to determine the currents. Since the field lines are almost vertical in the auroral region, the induction effect is much reduced in the latter estimates. Let us assume, for a first order estimate, a vertical field geometry and employ a line current at $110 \mathrm{~km}$ altitude. The current, $I$, in the line is estimated from the gradient of the vertical field component just above the line current according to Eq. (1) as:

$I=\frac{2 \pi h^{2}}{\mu_{o}} \frac{d b_{z}}{d x}$

where $\frac{d b_{z}}{d x}$ is the spatial derivative of the vertical component (here identical to the field magnitude) taken at the point above the line current.

When assuming a perfectly conducting sphere below $200 \mathrm{~km}$ depth we obtain a mirror current at $510 \mathrm{~km}$ depth flowing in the opposite direction to the ionospheric current. With an average satellite an altitude of $440 \mathrm{~km}$ the distance to the ionosphere is $330 \mathrm{~km}$ and to the mirror current $950 \mathrm{~km}$. Since the gradient of the vertical component, $\frac{d b_{z}}{d x}$, scales with $1 / h^{2}$, the mirror current will cause a reduction in the current estimate by $12 \%$. For the same configuration the ground data will experience an overestimation by $22 \%$ when using the horizontal components for the current determination, as has been done here. This demonstrates that the chosen induced current configuration has an effect on the estimates from the ground which is twice as strong as that from the CHAMP measurements. By comparing the results from the simple model with the difference of 10 to $15 \%$ derived by our correlation analysis we may conclude that the average induction effect associated with auroral electrojet in the IMAGE region is, on average, smaller. This is consistent with the findings of Baumjohann and Kamide (1981). 
After having verified and calibrated our method of the Hall currents estimate from satellite magnetic field data we would like to point out some areas of application. CHAMP is orbiting the Earth almost 16 times per day, each time crossing the polar regions in both hemispheres. From the current estimates an auroral activity index similar to $A E$ may be derived (cf. Fig. 2). The advantage of the satellite-derived index would be that it could include the current intensity, as well as the latitude and width of the auroral electrojet for both hemispheres. These quantities are important when monitoring the auroral activity. Although the magnetic activity recorded by CHAMP is, in general, well represented by the $A E$ index, there are significant differences evident in Fig. 2. For example, the very active period on MJD 302 is not appropriately reflected by $A E$. The reason for that is probably the large expansion of the polar cap during that event. As a consequence, the $A E$ stations miss major disturbances. The employment of spaceborne measurements becomes even more attractive when combining several low-Earth orbiting satellites. Using data from the presently active spacecraft Ørsted, CHAMP and SAC-C will allow us to monitor the auroral activity in both hemispheres very closely (e.g. Moretto et al., 2002).

The results obtained here are also of interest for the modelling of the internal part of the geomagnetic field from satellite measurements. Generally, only the field magnitude is used for modelling purposes at high latitudes. In this area the ionospheric Hall currents are the largest source of perturbations. As has been demonstrated here, these currents can be determined from ground and subsequently, the perturbation of the field magnitude can be predicted at satellite altitude. From our correlation analysis we obtained an uncertainty in the current estimate of $40 \mathrm{~mA} / \mathrm{m}$. According to Eq. (1) this converts into an uncertainty in the magnetic deflection of $3 \mathrm{nT}$ at CHAMP altitude. Having cleaned the data to that level will help enormously to derive reliable model results for the polar regions.

\section{Conclusions}

We have presented estimates of the ionospheric Hall currents derived independently from satellite and ground-based magnetic field observations. It turned out that total magnetic field data of low-Earth obit spacecraft can effectively be used to determine this current component. Equally, the spherical elementary current system (SECS) method showed its potential to estimate reliably the 2-D equivalent ionospheric current distribution from ground-based observations. By employing the results from both facilities in a systematic survey over 22 months we could identify the average characteristics of the auroral electrojet. Some of the derived features are:

- The most important controlling factor for the average activity is the solar zenith angle. This is particularly evident on the dayside.
- During the winter months, discrete storm/substorm events clearly stand out from the generally low background activity on the nightside.

- The activity enhancement due to the Russell-McPherron effect during equinox seasons is primarily confined to the night-time sector.

The excellent agreement between ground and satellite results has quantitatively been demonstrated in a correlation analysis. Whenever the current densities are above the detection level of $40 \mathrm{~mA} / \mathrm{m}$, significant correlations were obtained. Here we have provided for the first time a calibration and verification of the employed current determination from space. The differences in the obtained current intensities have been interpreted in terms of induction effects.

A useful application one could think of would be to employ the SECS method for estimating the ionospheric current distribution for periods of magnetic main field modelling and select suitable time intervals accordingly. In a next step, one could even predict the magnetic signature at the satellite, due to the good agreement demonstrated in our analysis and correct the ionospheric effect before modelling the data.

Acknowledgement. We thank all institutes maintaining the IMAGE magnetometer network. The operational support of the CHAMP mission by the German Aerospace Center (DLR) and the financial support for the data processing by the Federal Ministry of Education (BMBF) are gratefully acknowleged. The Academy of Finland supported the work of OA and AP. The DFG supported PR through the Priority Programme "Geomagnetic Variations", SPP 1097. The $A E$ index data were kindly provided by the WDC-C2 in Kyoto.

Topical Editor M. Lester thanks F. Christiansen and another referee for their help in evaluating this paper.

\section{References}

Amm, O. and Viljanen, A.: Ionospheric disturbance magnetic field continuation from the ground to the ionosphere using spherical elementary current systems, Earth Planets Space, 51, 431-440, 1999.

Baumjohann, W. and Kamide, Y.: Joint two-dimensional observations of ground magnetic and ionospheric electric fields associated with auroral zone currents: 2 . Three-dimensional current flow in the morning sector during substorm recovery, J. Geomagn. Geoelectr., 33, 297-318, 1981.

Fukushima, N.: Generalized theorem for no ground magnetic effect of vertical currents connected with Pedersen currents in the uniform-conductivity ionosphere, Rept. Ionos. Space Res. Japan, 30, 35-40, 1976.

Glassmeier, K.-H.: On the influence of ionospheres with nonuniform conductivity distribution by hydromagnetic waves, J. Geophys., 54, 125, 1984

Holme, R., Olsen, N., Rother, M., and Lühr, H.: CO2 - A CHAMP magnetic field model, in "First CHAMP Mission Results for Gravity, Magnetic and Atmospheric Studies", edited by Reigber, Lühr, and Schwintzer, Springer Berlin, 220-225, 2003.

Iijima, T. and Potemra, T. A.: The amplitude distribution of filedaligned currents at northern high latitudes observed by TRIAD, J. Geophys. Res., 81, 2165-2174, 1976. 
Kamide, Y. and Richmond, A. D.: Ionospheric conductivity dependence of electric fields and currents estimated from ground magnetic observations, J. Geophys. Res., 87, 8331-8337, 1982.

Lühr, H., Aylward, A., Buchert, S. C., Pajunpää, A., Pajunpää, K., Holmboe, T., and Zalewski, S. M.: Westward moving dynamic substorm features observed with the IMAGE magnetometer network and other ground-based instruments, Ann. Geophysicae, 16, 425-440, 1998.

Maus, S., Rother, M., Holme, R., Lühr, H., Olsen, N., and Haak, V.: First scalar magnetic anomaly map from CHAMP satellite data indicates weak lithospheric field, Geophys. Res. Lett., 29(14), 10.1029/2001GL013685, 2002.

Mersmann, U., Baumjohann, W., Küppers, F., and Lange, K.: Analysis of an Eastward Electrojet by Means of Upward Continuation of Ground-Based magnetometer Data, J. Geophys., 45, $281-$ $298,1979$.

Moretto T., Olsen, N., Ritter, P. and Lu, G.: Investigating the auroral electrojets with low altitude polar orbiting satellites, Ann. Geophysicae, 20, 1049, 2002.

Olsen, N.: A new tool for determining of ionospheric currents from satellite data, Geophys. Res. Lett., 23 3635-23 3638, 1996.
Olsen N.: A model of the geomagnetic main field and its secular variation for epoch 2000 estimated from Ørsted data, Geophys. J. Int., 149, 454-462, 2002.

Pulkkinen, A., Amm, O., Viljanen, A., and BEAR Working Group: Ionospheric equivalent current distributions determined with the method of spherical elementary current systems, J. Geophys. Res., 108(A2), 10.1029/2001JA005085, 2003.

Russell, C. T. and McPherron, R. L.: Semiannual variation of geomagnetic activity, J. Geophys. Res., 78, 92, 1973.

Sillanpää, I., Lühr, H., Viljanen, A., and Ritter, P.: Quiet-time Magnetic Variations at High Latitude Observatories, Earth Planets Space, (submitted), 2003.

Tanskanen, E. I., Viljanen, A., Pulkkinen, T. I., Pirjola, R., Häkkinen, L., Pulkkinen, A., and Amm, O.: At substorm onset, $40 \%$ of AL comes from underground, J. Geophys. Res., 106, 13 119-13 134, 2001.

Untiedt, J. and Baumjohann, W.: Studies of polar current systems using the IMS Scandinavian magnetometer array, Space Sci. Rev., 63, 245-390, 1993.

Zanetti, L. J., Baumjohann, W., and Potemra, T. A.: Ionospheric and Birkeland current distributions inferred from the MAGSAT magnetometer data, J. Geophys. Res., 88, 4875-4884, 1983. 